\title{
Personalia: Wechsel im Editorial Board der Klinischen Pädiatrie
}

\section{Peronalia - Changes in the Editorial Board of the Klinische Pädiatrie}

Ludwig Gortner

Bibliografie

DOI http://dx.doi.org/

10.1055/s-0042-114822

Klin Padiatr 2016; 228: 285

(c) Georg Thieme Verlag KG

Stuttgart · New York

ISSN 0300-8630

Korrespondenzadresse

Ludwig Gortner, Herder-

Professor

Klinik für Kinder-und

Jugendheilkunde

Medizinische Universität Wien

Währinger Gürtel 18-20

1090 Wien

Österreich
Zum Beginn des 3. Quartals des laufenden Jahres haben sich 2 Veränderungen im Bereich der der Herausgeber und der verlagsseitigen Betreuung der Klinischen Pädiatrie ergeben, die Gegenstand des Editorials sein sollen:

Zum einen hat Herr Prof. D. Körholz die Tätigkeit als Managing Editor der Klinischen Pädiatrie zu diesem Zeitpunkt abgegeben, diese Funktion seit Anfang des Jahres 2013 inne. In dem genannten Zeitraum war es mir eine Freude und Ehre, gemeinsam als Herausgeber dieser Zeitschrift mit ihm zu arbeiten. In dieser Periode wurden bedeutsame Publikationen, welche eine hohe internationale Zitationsquote haben, und die für Tendenzen innerhalb der klinischen Versorgung bedrohlich Erkrankter relevant waren, publiziert. Es seien bespielhaft genannt unter anderem das Konsensus-Statement zur Knochenmarkstransplantation bei Patienten mit Fanconi-Anämie [1] und Behandlungsempfehlungen zu spezifischen onkologischen Subgruppen des Hodgkin-Lymphoms [2]. Die Bedeutung der Publikationen wird durch die Häufigkeit der Zitationen unterstrichen. Spezifische Betrachtungen zur Diagnostik und Therapie seltener Erkrankungen, welche nicht nur in der Pädiatrie eine zunehmend in den Fokus des wissenschaftlichen und klinischen Interesses geratene Entität darstellen, ergänzen die von Herrn Prof. Körholz gesetzten thematischen Schwerpunkte [3].

Die zitierten Original- und Übersichtsarbeiten mögen belegen, dass das wissenschaftliche und klinische Profil der Zeitschrift innerhalb der letzten 3 Jahre ganz wesentlich durch die Aktivität von Herrn Prof. Körholz geprägt wurde. Hierfür darf ich ihm meinen ganz herzlichen Dank aussprechen!

Die Bemühungen der Herausgeber wären jedoch insgesamt weniger effizient oder gänzlich zum Scheitern verurteilt, wenn es keine engagierte Betreuung der Zeitschrift seitens des Verlages gäbe. Ohne zu viele Details beim Blick hinter die Kulissen des verlagsseitigen Engagements zu geben, mögen hier beispielhaft für die Aktivitäten von Herrn V. Niem, welcher die Zeitschrift vom Jahre 2002 bis zur Mitte des Jahres 2016 betreute, aufgeführt werden:

Innerhalb dieses Zeitraumes hat sich die Klinische Pädiatrie trotz mancher Diskussion, im Verlag, welche auch die Herausgeber erreichte, weiter zu einer festen Größe in dem Zeitschriftenportfolio entwickelt. Auf der technischen Ebene fällt die elektronische Verwaltung der Manu- skripte, welche im Jahre 2008 eingeführt wurde in diese Zeit. Dies war neben den Bemühungen der Herausgeber eine der Grundlagen für die Internationalisierung der Zeitschrift. Der mittlerweile recht konstant gehaltene Anteil von englischsprachigen Manuskripten im Bereich von 40 bis $50 \%$ wäre ohne die genannten Aktivitäten sicherlich ebenso schwierig zu halten, wie der Impact Faktor der Zeitschrift. Dieser hat sich zwischen 1 und 1,1 während der letzten beiden Jahre bewegt.

Das Konzept der Internationalisierung der Zeitschrift hat in der Landschaft der deutschen pädiatrischen Zeitschriften neben reinen Weiterbildungsjournalen mit nahezu ausschließlich deutschsprachigen Manuskripten sowie einer rezent eingeführten Zeitschrift mit im Wesentlichen englischsprachig gehaltenen basis-wissenschaftlichen Inhalten, die Intermediärposition der Klinischen Pädiatrie für die klinische Forschung gesichert.

Zuletzt - last but not least - darf ich als neuen Mitherausgeber Herrn Prof. C. Kratz aus der Medizinischen Hochschule Hannover ganz herzlich begrüßen, Dank schon jetzt für seine Bereitschaft, insbesondere den bewährten onkologischen Schwerpunkt der Klinischen Pädiatrie weiter zu sichern und auszubauen. Das neue Herausgeberteam freut sich auf die Zusammenarbeit mit Fr. Dr.Kapousouzi., die verlagsseitig die Klinische Pädiatrie weiter betreuen wird.

Der abschließende Dank und Anerkennung gebührt den Herrn Prof. Körholz sowie Niem für ihre Aktivitäten im Rahmen der Fortentwicklung der Klinischen Pädiatrie.

\section{Literatur}

1 Chao MM, Ebell W, Bader P et al. Consensus of German transplant centers on hematopoietic stem cell transplantation in Fanconi anemia. Klin Padiatr 2015; 227: 157-165

2 Mauz-Korholz C, Lange T, Hasenclever D et al. Pediatric Nodular Lymphocyte-predominant Hodgkin Lymphoma: Treatment Recommendations of the GPOH-HD Study Group. Klin Padiatr 2015; 227: 314-321

3 Korholz $D$, Schneider $D$, von Kries $R$ et al. How to Overcome Scientific Standstill for Very Rare Diseases: Clinical Trials or Clinical Registries? Klin Padiatr 2015; 227: 303-304 